\title{
The thalamus and multiple sclerosis
}

\author{
Modern views on pathologic, imaging, and clinical aspects
}

Alireza Minagar, MD

Michael H. Barnett, MD, $\mathrm{PhD}$

Ralph H.B. Benedict, $\mathrm{PhD}$

Daniel Pelletier, MD

Istvan Pirko, MD

Mohamad Ali Sahraian, MD

Elliott Frohman, MD, $\mathrm{PhD}$

Robert Zivadinov, MD, $\mathrm{PhD}$

Correspondence to

Dr. Zivadinov:

rzivadinov@bnac.net

\section{ABSTRACT}

The paired thalamic nuclei are gray matter (GM) structures on both sides of the third ventricle that play major roles in cortical activation, relaying sensory information to the higher cortical centers that influence cognition. Multiple sclerosis (MS) is an immune-mediated disease of the human CNS that affects both the white matter (WM) and GM. A number of clinical observations as well as recent neuropathologic and neuroimaging studies have clearly demonstrated extensive involvement of the thalamus, basal ganglia, and neocortex in patients with MS. Modern MRI techniques permit visualization of GM lesions and measurement of atrophy. These contemporary methods have fundamentally altered our understanding of the pathophysiologic nature of MS. Evidence confirms the contention that GM injury can be detected in the earliest phases of MS, and that iron deposition and atrophy of deep gray nuclei are closely related to the magnitude of inflammation. Extensive involvement of GM, and particularly of the thalamus, is associated with a wide range of clinical manifestations including cognitive decline, motor deficits, fatigue, painful syndromes, and ocular motility disturbances in patients with MS. In this review, we characterize the neuropathologic, neuroimaging, and clinical features of thalamic involvement in MS. Further, we underscore the contention that neuropathologic and neuroimaging correlative investigations of thalamic derangements in MS may elucidate not heretofore considered pathobiological underpinnings germane to understanding the ontogeny, magnitude, and progression of the disease process. Neurology ${ }^{\circledR} 2013 ; 80: 210-219$

\section{GLOSSARY}

CIS = clinically isolated inflammatory demyelinating syndrome; DIR = double inversion recovery; DTI = diffusion tensor imaging; EDSS = Expanded Disability Status Scale; GM = gray matter; LGN = lateral geniculate nucleus; $\mathbf{M S}=$ multiple sclerosis; NAGM = normal-appearing gray matter; NAWM = normal-appearing white matter; $\mathbf{S W I}=$ susceptibility-weighted imaging; $\mathbf{W M}=$ white matter

Multiple sclerosis (MS) is a progressive inflammatory and degenerative disease of the human CNS that leads to demyelination and neuronal/axonal loss. Both the etiology and cure for MS remain elusive, and for many years scientific research into the pathogenesis of MS has heavily focused on a disease principally affecting CNS white matter (WM).

Notwithstanding the traditional focus upon WM as the predominant target of the disease mechanisms in MS, recent findings, which indicate significant gray matter (GM) involvement, are an important and substantial refinement in our understanding of the pathobiological underpinnings of the disease process in MS, of particular relevance to cognitive decline as well as overall disease worsening. ${ }^{1,2}$ Neuropathologic data implicate significant cortical demyelination and neuro-axonal and synaptic loss in both the early and late phases of the disease process. ${ }^{2-5}$ Both cortical ${ }^{3}$ and subcortical demyelination are observed during the course of MS, targeting a landscape of GM-rich structures including the thalamus, hippocampus, caudate, putamen, globus pallidus, and other structures of the basal ganglia.,

Both postmortem and in vivo studies have revealed extensive MS lesions of the GM structures. ${ }^{17,8}$ In general, the neuropathologic features of GM involvement in MS differ significantly

From the Department of Neurology (A.M.), Louisiana State University Health Sciences Center, Shreveport, LA; Department of Neurology (M.H. B.), Royal Prince Alfred Hospital, Sydney; Brain \& Mind Research Institute (M.H.B.), University of Sydney, Australia; The Jacobs Neurological Institute (R.H.B.B., R.Z.) and Buffalo Neuroimaging Analysis Center, Department of Neurology (R.Z.), University at Buffalo, Buffalo, NY; Departments of Neurology and Diagnostic Radiology (D.P.), Yale University, New Haven, CT; Department of Neurology (I.P.), Mayo Clinic, Rochester, MN; Sina MS Research Center (M.A.S.), Sina Hospital, Tehran University of Medical Sciences, Tehran, Iran; and Department of Neurology (E.F.), UT Southwestern Medical Center, Dallas, TX.

Go to Neurology.org for full disclosures. Funding information and disclosures deemed relevant by the authors, if any, are provided at the end of the article. 
from WM lesions. ${ }^{5}$ For example, certain features such as lymphocyte infiltration, complement deposition, and disruption of the blood-brain barrier are typically not detected in the GM of chronic autopsy samples but have been reported in cortical lesions of active MS. More robust and long-lasting neuroinflammation is the dominant component of newly forming WM lesions. Similar to diffuse involvement of the WM in MS, demyelination of the cerebral cortex and cerebellum, ${ }^{1}$ deep GM structures, ${ }^{8,9}$ and the GM of the spinal cord ${ }^{10}$ have been reported, supporting the concept of "diffuse brain involvement" in MS. Pathology within the thalamus is frequently observed in MS, but less well characterized when compared to the delineation of WM compartment lesions. ${ }^{7,8}$ As a major portion of the diencephalon in primates, the thalamus serves as a gateway to the cerebral cortex and is heavily involved with its activity. However, its unique structural division into various nuclear complexes enables the thalamus to connect with other subcortical structures. Neuroanatomically, as a "relay organ," the thalamus is involved in a wide range of neurologic functions including motor, sensory, integrative, and higher cortical functions. Further, the thalamus also plays a significant role in other functions such as the regulation of sleep and wakefulness, memory, emotion, consciousness, awareness, and attention. The thalamus is also involved in ocular motility, posture, and executive function. Location, unique functions, and vulnerability to MS neuropathology from the earliest disease stages ${ }^{11-14}$ render the thalamus a critical barometer of diffuse neuropathologic damage in MS. Advanced neuropathologic and neuroimaging methods are slowly emerging, providing us with a better view of the full extent of thalamic abnormalities in patients with MS.

Thalamic axons are well known to transmit information between a number of subcortical and specific cortical areas. As such, damage to the thalamic nuclei and their connections potentially impairs a wide range of neurologic functions that may clinically translate into significant cognitive and mental disability. ${ }^{15,16}$ Thalamic pathology in MS accumulates on a background of normal age-related reduction in thalamic volume, ${ }^{17}$ which is in itself associated with a decline in cognitive capacity, particularly mental speed and information processing speed. ${ }^{18}$ Predicated on the known role of the thalamus in the normal operation of the human CNS, and based on the growing knowledge of the extent of its damage and involvement in the pathogenesis of MS, we aimed to review and discuss these abnormalities in the context of recently emerging neuropathologic and neuroimaging studies.

THALAMIC NEUROPATHOLOGY IN MS The notion that clinically relevant MS pathology is restricted to focal WM lesions has been overwhelmingly negated by an expanding body of neuropathologic data implicating significant cortical myelin, neuro-axonal, and synaptic loss $^{2-5,9,19}$ in both early and late stages of the disease. Pathology afflicting the deep GM structures, in particular the thalamus, is frequently observed in MS, but less well studied. Significant neuropathologic and neuroimaging studies addressing thalamic involvement in MS are presented in the table. While informative, these studies are limited by their small sample size, retrospective design, registration biases, and an absence of treatment effects on thalamic MRI-related outcomes over the course of time.

Distribution. The extent of subcortical GM demyelination correlates well with the extent of cortical, but not WM, demyelination, ${ }^{8}$ although more studies are needed to determine the relationship between the pathologic processes operating in the subcortical GM with respect to those in the WM. Thalamic GM lesions may extend into the surrounding WM; however, a significant proportion of deep gray plaques are in fact confined to the subcortical GM, with edges that are abruptly demarcated in juxtaposition with well-recognized anatomic WM borders. ${ }^{810}$ Extensive, confluent subependymal GM demyelination is relatively common in the paraventricular nuclei of the thalamus, ${ }^{10}$ raising the possibility of a pathogenic factor within the CSF. While this hypothesis is supported by the demonstration of B-cell aggregates in association with the fourth ventricle and its lateral recesses in the experimental allergic encephalomyelitis model of MS, ${ }^{20}$ the "follicles" identified in the meninges in topographic association with zones of subpial demyelination in the cortex of some patients with secondary progressive disease ${ }^{21}$ have not been identified in association with the ventricular system in humans.

Diffuse microglial activation is detectable in thalamic normal-appearing GM (NAGM), ${ }^{8}$ but to a lesser degree than one typically observes in the normal-appearing WM (NAWM) of patients with long-standing disease. Extralesional microglial activation, characterized by thickening of cell processes and upregulation of human leukocyte antigen-DR expression, is a highly sensitive, albeit nonspecific, indicator of CNS pathology. In MS, retrograde and anterograde diffuse axonal injury is the 
Original article

Cifelli et al. (2002) ${ }^{7}$

No. of subjects and disease subtype

14 SPMS and 14 controls

Wylezinska et al. (2003) $)^{51}$

14 RRMS and 14 controls

Vercellino et al. (2005) ${ }^{9}$

6 MS (3 RRMS and 3 SPMS)

Davies et al. (2004) $)^{47}$

38 RRMS and 35 controls

Houtchens et al. (2007) ${ }^{16}$

79 MS (62 RRMS, 16 SPMS, and 1 PPMS) and 16 controls

Mesaros et al. (2008) $)^{37}$

28 pediatric RRMS and 21 controls

Vercellino et al. (2009) ${ }^{8}$

Ramasamy et al. (2009) ${ }^{13}$

Gilmore et al. (2009) ${ }^{10}$

Henry et al. (2009) 11

Tovar-Moll et al. (2009) 49

24 MS (13 RRMS and 11 SPMS) and 24 controls

Rocca et al. (2010) ${ }^{15}$

73 relapse-onset MS (20 CIS, 34 RRMS, and 19 SPMS) and 13 controls

$62 \mathrm{CIS}$ and 37 controls

Audoin et al. (2010) $)^{33}$

Calabrese et al. (2011) ${ }^{14}$

$105 \mathrm{CIS}$ and 24 controls

Mesaros et al. (2011) ${ }^{48}$

54 PPMS and 8 controls

Hagemeier et al. (2012) ${ }^{41}$

$42 \mathrm{CIS}$ and 65 controls

Batista et al. (2012) $)^{59}$

86 MS (59 RRMS and 27 SPMS) and 25 controls

Zivadinov et al. (2012) $)^{44}$ controls

\section{Major neuropathologic and neuroimaging findings}

Utilizing MRI, MRS, and postmortem histopathologic studies with focus on thalamic neuronal loss, the authors reported significant neuronal damage in MS.

Utilizing structural MRI and MRS studies, the authors assessed thalamic neurodegeneration in patients with RRMS and reported reduction of both NAA concentration and thalamic volume, which indicated a neurodegeneration component in the neuropathology of MS. The authors concluded that the decrease in NAA concentration and thalamic volume suggests that neurodegeneration contributes to the neuropathology of MS in an even earlier stage of the disease.

The authors performed a neuropathologic examination of MS brains to assess the extent and distribution of GM demyelinating lesions in MS with a focus on neuronal loss and synaptic loss. They reported the presence of demyelinating lesions in the thalamus, cerebral cortex, basal ganglia, and hippocampus. It was concluded that GM demyelination and neuronal loss could contribute to disability and cognitive decline in MS.

Utilizing MTR in patients with early RRMS, the authors reported abnormality of thalamic MTR and thalamic involvement within the first 5 years of MS onset.

Normalized thalamic volume was lower in patients with MS compared to controls, and cognitive performance in all domains was related to thalamic volume in the MS group. A weak correlation between thalamic atrophy and physical disability score was detected. However, cognitive performance in all domains was moderately to strongly related to thalamic volume in patients with MS. The authors concluded that thalamic atrophy was a clinically relevant biomarker of the neurodegenerative disease process in MS.

In pediatric patients with MS, different from what occurs in adult patients with MS $\mathrm{GM}$ atrophy appears to affect the thalamus exclusively.

Demyelination and neurodegenerative changes are common in MS deep GM and may contribute to clinical deficits.

This study supported selective deep GM atrophy (mostly thalamic), showed cerebellar WM atrophy from the earliest clinical stages, and showed that cortical thinning advances with disease progression.

The authors reported the presence of significant regional variations in the extent and pattern of GM demyelination in MS.

A stepwise regression analysis demonstrated that thalamocortical lesion volume and the mean diffusivity in track regions connecting lesion and thalami were significantly correlated with thalamic volumes in patients, explaining $66 \%$ of the variance, a finding not observed in regions outside the thalamocortical WM.

Utilizing DTI at 3 T, the authors assessed thalamic involvement and its impact on clinical disability in patients with MS. They concluded that DTI was able to detect abnormalities in the normal-appearing thalamus of patients with MS and there was an association between thalamic DTI measures and functional impairment in MS.

Utilizing conventional imaging and MTR, the authors detected that thalamic atrophy was correlated with accumulation of disability in patients with MS. In addition, WM lesions were likely to contribute to the thalamic tissue loss observed in patients with MS.

Based on this study which involved patients with CIS, atrophy mainly affected the limbic system and the deep GM (bilateral thalami) at the first stage of MS.

The study found that selective GM atrophy is relevant in patients with CIS who convert early to MS. The multivariate analysis identified the atrophy of the superior frontal gyrus, thalamus, and cerebellum as independent predictors of conversion to MS.

Using conventional and DTI brain scans, the authors assessed thalamic damage in patients with PPMS and concluded that thalamic damage predicts the evolution of PPMS.

Patients with CIS showed significantly increased content and volume of iron, as determined by abnormal SWI-phase measurement in the various SDGM structures, especially in the pulvinar nucleus of thalamus, suggesting that iron deposition may precede structure-specific atrophy.

Utilizing $3 \mathrm{~T}$ brain MRI, the authors demonstrated that the basal ganglia, thalamus, and neocortical atrophy predict slowed cognitive processing in MS.

Utilizing SWI-filtered phase images, the authors assessed subcortical GM structure in patients with MS compared to control subjects, and reported abnormal phase in patients with MS in a number of GM regions, especially in the pulvinar nucleus of thalamus, indicative of higher iron content in patients with MS, which was related to more severe lesion burden and brain atrophy.

Abbreviations: ALS = amyotrophic lateral sclerosis; CIS = clinically isolated syndrome; DTI = diffusion tensor imaging; GM = gray matter; MRS = magnetic resonance spectroscopy; MS = multiple sclerosis; MTR = magnetization transfer ratio; NAA = N-acetylaspartate; PPMS = primary progressive multiple sclerosis; RRMS = relapsing-remitting multiple sclerosis; SDGM = subcortical deep gray matter; SPMS = secondary progressive multiple sclerosis; $\mathrm{SWI}=$ susceptibilityweighted imaging; $\mathrm{WM}=$ white matter. 
Figure 1 Coronal section through the posterior thalamus, secondary progressive multiple sclerosis

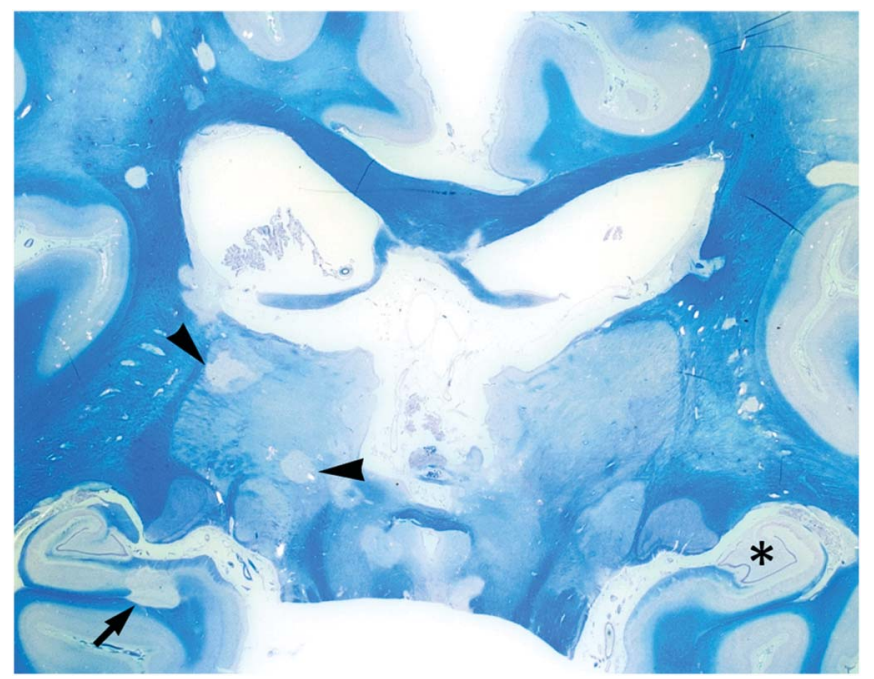

Focal chronic lesions are present in the right pulvinar nuclei and the lateral posterior nucleus of the thalamus (arrowheads), the latter with a remyelinating fringe. Demyelination is also present in the deep gray matter of the right caudate nucleus in association with an adjacent chronic white matter lesion. Additionally, there is a leukocortical lesion in the subiculum of the right hippocampal formation (arrow), diffuse demyelination of the left hippocampus proper (asterisk), and involvement of the periaqueductal gray matter. Classic small focal white matter lesions are present in the white matter, including the corpus callosum. Luxol fast blue.

likely substrate underlying this finding in both NAWM and NAGM, although diffusion of soluble cytokines from nearby focal lesions, or a primary innate immune response, are possible contributors.

Pathology of focal lesions. Histopathologic characterization of the thalamic lesions (figure 1) recapitulates the

\section{Figure 2 Border zone of a chronic thalamic lesion, secondary progressive multiple sclerosis}

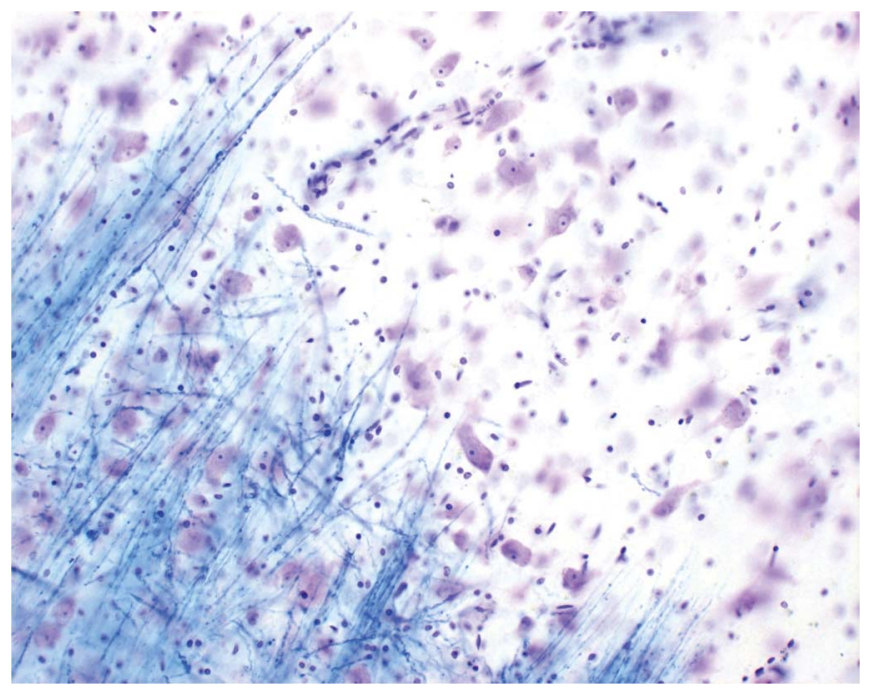

The gray matter lesion (top right) is devoid of myelin (stained blue) and hypocellular. Comparison with adjacent normal-appearing gray matter (bottom left) discloses a reduction in the number of neurons and a paucity of cells with oligodendrocyte morphology (Luxol fast blue). spectrum of active, chronically demyelinated, and remyelinated pathologies observed in the WM. Chronic lesions, completely devoid of myelin (figure 2), predominate in the few existing pathologic descriptions, and exhibit a relatively subdued astrocyte reaction as indicated by glial fibrillary acid protein immunohistochemistry. ${ }^{8}$ Within rare active lesions, the intensity of the macrophage infiltrate mirrors myelin density and therefore varies according to location within the thalamus. Similar to changes demonstrated in cortical GM lesions of autopsy cases, ${ }^{22-24}$ both adaptive ( $\mathrm{T}$ and $\mathrm{B}$ cell perivascular and parenchymal infiltration) and innate (microglial activation/macrophage infiltration) inflammation in the thalamus is constrained when compared with classic active WM lesions, ${ }^{8}$ a factor that potentially limits neuroaxonal injury in evolving lesions. However, this view has been recently challenged by Lucchinetti et al., ${ }^{3}$ who demonstrate significant cortical GM inflammation in biopsy cases of early MS. Sampling bias in both early biopsy and end-stage autopsy studies may explain these discrepant findings; larger neuropathologic studies of the subcortical GM structures are required in order to corroborate or refute these observations.

Neuronal pathology. Thalamic pathology in MS cannot be viewed in isolation. As a discrete, symmetric brain structure with widespread cortical and subcortical connections, the thalamus provides the ideal neuropathologic (and MRI) substrate for examining neurodegeneration in MS. Cifelli et al. ${ }^{7}$ examined the thalami of 10 patients with MS (9 progressive, 1 relapsing) and demonstrated a reduction in both thalamic volume (21\%) and nonlesional neuronal density $(22 \%)$ vs age- and gender-matched controls, confirming their in vivo MRI observations in a separate cohort of patients with secondary progressive MS. A similar degree of neuronal loss was subsequently described in nonlesional mediodorsal thalamic nucleus, ${ }^{8}$ raising the possibility that neuronal loss is a retrograde event consequent to axonal transection in WM tracts projecting from the thalamus, or secondary to transsynaptic deafferentation of thalamic neurons. Altered neuronal morphology has also been described in the lateral geniculate nucleus (LGN) of the thalamus, ${ }^{25}$ which receives projections from axons in the optic tract. The authors describe significant variation and an overall reduction in neuronal size in the parvocellular, but not magnocellular, layers of the LGN, which they attribute to retrograde damage resulting from differential susceptibility of small diameter axons (in the optic tract) to MS-related injury. It seems unlikely that Wallerian or transsynaptic degeneration is the sole cause of neuronal damage in the thalamus. Direct injury from diffusable cytokines, oxidative stress, excitotoxicity, and CD8 T-cell-mediated cytotoxicity are all contenders, particularly in cases with identifiable focal inflammatory lesions. Alteration in neuronal 
morphology and size within demyelinated thalamic GM lesions compared with adjacent nonlesional GM supports this hypothesis. ${ }^{8}$ Finally, surviving neurons within subcortical GM lesions invariably shed their satellite oligodendrocytes, potentially diminishing trophic support and disrupting the perineuronal microenvironment. In particular, perineuronal glutamate homeostasis may be dysregulated by the loss of glutamate transporterexpressing satellite oligodendrocytes. ${ }^{24}$

NEUROIMAGING CHARACTERISTICS OF THALAMIC INVOLVEMENT IN MS Due to inherent structural differences between GM and WM and as a result of differences in inflammation characteristics, cortical GM lesions seem to maintain a normal water proton concentration and, unlike WM lesions, are not typically detectable as T2 hyperintense foci on MRI. ${ }^{26}$ However, thalamic lesions are usually more visible than cortical lesions, likely because the thalamus is normally more densely myelinated. The introduction of double inversion recovery (DIR) in the study of MS may play an important step in refining the conspicuity of GM lesions. ${ }^{6}$ By suppressing the signal from normal WM and CSF, DIR sequences provide excellent differentiation between GM and WM. ${ }^{27}$ In a series of recently published studies from the Amsterdam (Netherlands) ${ }^{6}$ and Padova (Italy) ${ }^{28}$ groups, DIR imaging most frequently identified cortical lesions in male patients, progressive MS, and in those with CSF IgG oligoclonal bands. However, it is now evident that DIR imaging detects only a fraction of the real burden of cortical GM pathology that is present in patients with MS, with an average sensitivity of only $18 \%{ }^{29}$ Therefore, although pathologically validated DIR scoring indicates a high specificity for the detection of GM lesions, the sensitivity is very low, with a false-positive rate of at least $10 \% .^{29,30}$ The value of DIR in the detection of lesions in subcortical GM lesions was not investigated.

Contemporary investigations have focused on determining the extent of cortical and subcortical GM pathology in patients presenting with a first clinically isolated inflammatory demyelinating syndrome (CIS), ${ }^{11-13,31-33}$ or following conversion to clinically definite MS. ${ }^{14,34}$ It has been reported that global and cortical GM volume measures are not sensitive enough to detect GM atrophy at the time of the initial attack. ${ }^{31}$ Therefore, GM atrophy studies in patients with CIS have increasingly turned to regional segmentation techniques to identify specific structures with a stronger predilection for disease susceptibility and conversion to clinically definite MS. A number of independent studies have shown that loss of volume in the thalamus is one of the earliest and most prominent signs of subcortical GM pathology when patients present with CIS. ${ }^{11-14,31,33,35}$ Progressive atrophy of the thalamus (figure 3 ) has been shown in all different MS disease types ${ }^{12,13,15,36,37}$ and thalamic volume loss has also been detected in pediatric patients with MS. ${ }^{37-40}$

In a recent study of 42 patients with CIS, it was demonstrated that deposition of iron, as estimated by susceptibility-weighted imaging (SWI), in the thalamus and the pulvinar nucleus of the thalamus may precede the development of structure-specific thalamic atrophy at MS clinical onset. ${ }^{41}$ This finding has to be confirmed in future studies; however, it is in keeping with mounting evidence derived from T2 hypointensity, ${ }^{42,43}$ SWI, ${ }^{44}$ and relaxometry ${ }^{45}$ studies that showed that iron overload is dominant in the thalamus of patients with MS. One can hypothesize that a vicious cycle involving proinflammatory cells, atrophy of the thalamus, and possible iron accumulation may occur throughout the course of MS. Characterizing the temporal and dynamic interplay of these potential pathobiologic mechanisms from the first clinical onset mandates further investigation; however, at this time there is no definitive answer for the role of iron in relation to thalamic damage in patients with MS.

A number of diffusion-weighted or tensor, ${ }^{11,46} \mathrm{mag}$ netization transfer, ${ }^{46-49}$ and magnetic resonance spectroscopy $7,50,51$ imaging studies have investigated the extent of thalamic damage in patients with MS with various disease types (table). All suggested that there may be a common mechanism for WM axonal loss and thalamic neuronal injury related to thalamocortical pathways. One study of 24 patients with CIS sought to determine whether the association between WM lesions and thalamic atrophy at first clinical onset is related to connectivity of these fibers. ${ }^{11}$ Diffusion tensor imaging (DTI) fiber tracking was used to create probabilistic templates of thalamocortical WM projections (figure 4A), and to define lesional thalamocortical WM tracks (figure 4B). There was a 10-fold higher density of lesions in thalamocortical projections compared to other brain WM regions. Using a stepwise linear regression model, DTI metrics within lesional thalamocortical tracks and thalamocortical lesion volume accounted for $66 \%$ of the variance in thalamic volume, while none of the MRI metrics outside thalamocortical regions contributed to thalamic volume. The authors suggested that focal MS lesions could cause distal WM injury and such damage could, at least in part, lead directly to thalamic neuronal and volume loss.

Taken together, these studies suggest that there is an urgent need for future longitudinal studies investigating the relationship between WM lesion accumulation, thalamic iron deposition, atrophy development, and microstructural alterations using nonconventional MRI metrics from the earliest clinical onset.

CLINICAL MANIFESTATIONS Clinically, thalamic involvement in MS manifests with a spectrum of diverse abnormalities, which range from fatigue and movement disorders to painful syndromes and cognitive decline. 

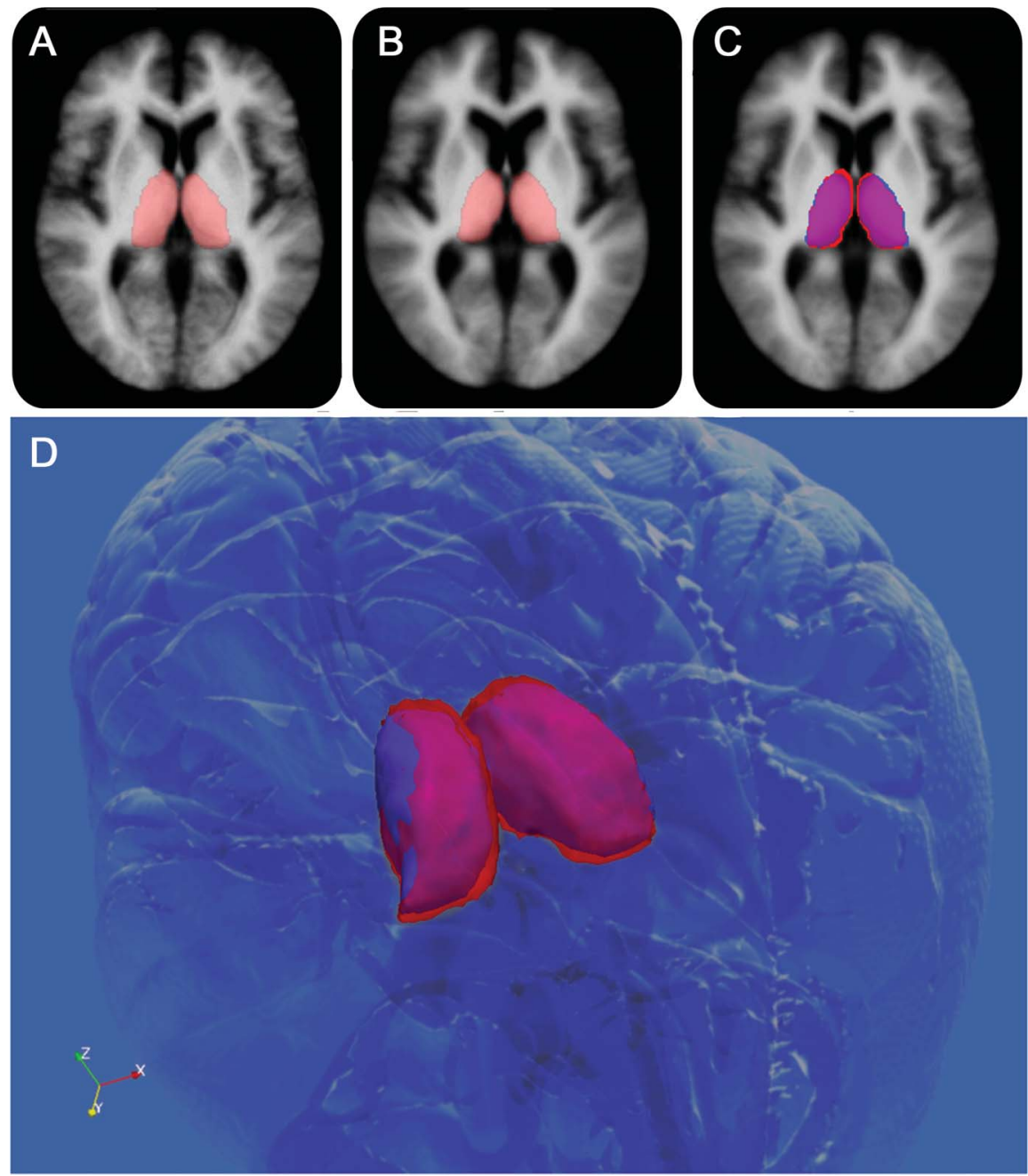

Comparison of thalamic segmentations between 26 healthy controls $(\mathrm{HC})$ (age- and sex-matched) ( $\mathrm{A}$ ) and 98 patients with relapsing-remitting multiple sclerosis (MS) (B). (C) Voxel-wise differences between the groups, with magenta representing common areas, red HC-only areas, and blue MS-only areas. Individual patient images were coregistered into the Montreal Neurological Institute 152 space for visualization. A 3D view (D) shows that the MS thalami are smaller overall and slightly shifted away from the midline (most likely by global atrophic processes).

Fatigue is perhaps the most common and disabling symptom of patients with MS and is defined as an overwhelming feeling of a lack of both mental and physical

Figure 4 Representative 3D rendering example of fiber tracking from diffusion tensor imaging and voxel-based morphometry in multiple sclerosis
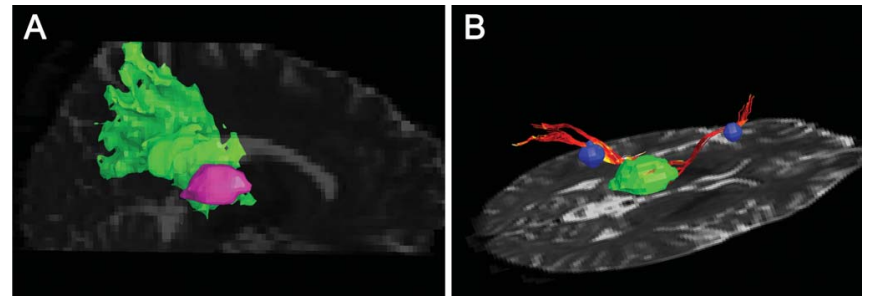

(A) Example of a probabilistic thalamoparietal template. The Montreal Neurological Institute transformed map is coregistered into the patient space; the thalamoparietal region of interest (ROI) is in green and the thalamus ROI in pink. (B) The figure shows 2 thalamocortical fiber tracks (red) seeded through white matter lesions (blue) connecting the thalamus (green) and the cortex (not shown). energy. Up to $80 \%$ of patients with MS are affected by fatigue, which interferes with a patient's quality of life. ${ }^{52}$ Despite significant effort to elucidate the pathogenic mechanisms of fatigue in patients with MS, our knowledge remains marginal, with many unanswered questions. Neuroimaging studies have significantly contributed to the analysis of the pathogenesis of fatigue in $\mathrm{MS}^{36,53}$ and have revealed functional abnormalities of neuroanatomic pathways involving frontal cortex, basal ganglia, and thalamus. ${ }^{36,54}$

A number of movement disorders have been described in association with MS, ${ }^{55}$ which include tremor, parkinsonism, myoclonus, chorea, and paroxysmal dystonia. Paroxysmal dystonia is a painful manifestation of MS that usually presents with unilateral dystonic posture, precipitated by voluntary movement, tactile stimuli, or hyperventilation. Zenzola et al. ${ }^{56}$ reported 2 patients with MS with paroxysmal dystonia and thalamic lesions. Both 
of these patients had developed demyelinating thalamic lesions contralateral to the paroxysmal symptoms.

Cognitive impairment is perhaps the most devastating and difficult to treat clinical manifestation of thalamic involvement in MS. In 2004, Benedict et al. ${ }^{57}$ investigated associations between conventional brain MRI metrics and cognitive function in patients with MS. Contrary to expectation, third ventricle width was most strongly associated with several validated neuropsychological tests. Noting the recent evidence of thalamic atrophy in MS, ${ }^{7}$ it was speculated that this finding was driven by atrophy (i.e., ex vacuo dilatation of the third ventricle) of the surrounding thalamic structures (figure 5). The hypothesis was directly investigated by tracing thalami from coronal $3 \mathrm{D}$ images, the boundaries being determined with an edge-finding tool and manual adjustment. ${ }^{16}$ The raw thalamic volumes were then normalized as a ratio to intracranial volume. Analysis revealed a 16.8\% reduction in volume in patients with MS compared to controls. In addition, thalamic fraction was the most significant MRI predictor of consensus standard ${ }^{58}$ tests of memory and processing speed. These results were recently replicated in a larger, independent sample using the FIRST technique. ${ }^{59}$ A similar theme has recently emerged in the pediatric MS literature. While Mesaros et al. ${ }^{60}$ found similar whole brain parenchyma and GM volumes between patients and controls, voxel-based morphometry analysis and statistical parametric mapping detected clusters of reduced GM concentration in the thalamus. Correlations with disease duration and the Expanded Disability Status Scale (EDSS) were not, however, significant. The authors acknowledged that EDSS does not adequately assess cognitive ability. When neuropsychological testing was applied to a pediatric sample, ${ }^{40}$ patients with MS again were found to have significantly lower thalamic volume. This time, however, volume loss was the most significant MRI predictor of cognition compared to other MRI metrics. As in the earlier adult literature, ${ }^{16,57}$ the degree of correlation was robust, with $r$ values ranging from 0.6 to 0.7 . A notable observation from both lines of research is that cognitive dysfunction in multiple spheres has been consistently associated with thalamic atrophy in children and adults with MS. In the pediatric study, ${ }^{40}$ the correlation between thalamus volume and IQ was $r=0.68$. The thalamic fraction or thalamic normalized volume represents a novel approach to quantifying regional GM volume, although it has to be acknowledged that the measure is crude from a neuropsychological perspective. The various nuclei in the thalamus have very different afferent and efferent connections to various brain regions and, as such, mediate disparate functions. Examining correlations with the anterior nucleus, which has rich connections with multiple frontal cortex areas, may shed more light on the link between thalamic pathology and neuropsychological involvement in MS.

DISCUSSION Cortical and subcortical GM involvement is a critically important contributor to the disease process in MS and leads to significant cognitive disability and other potentially devastating neurologic problems in these patients. Thalamic pathology, similar to the cortical pathology, appears to be present in MS from very early on, including at the CIS stage and in pediatric MS. In the progressive phase of MS, which is poorly explained by focal inflammatory WM demyelination, cortical and subcortical GM pathology including

Figure 5 Thalamic atrophy and third ventricle width in multiple sclerosis

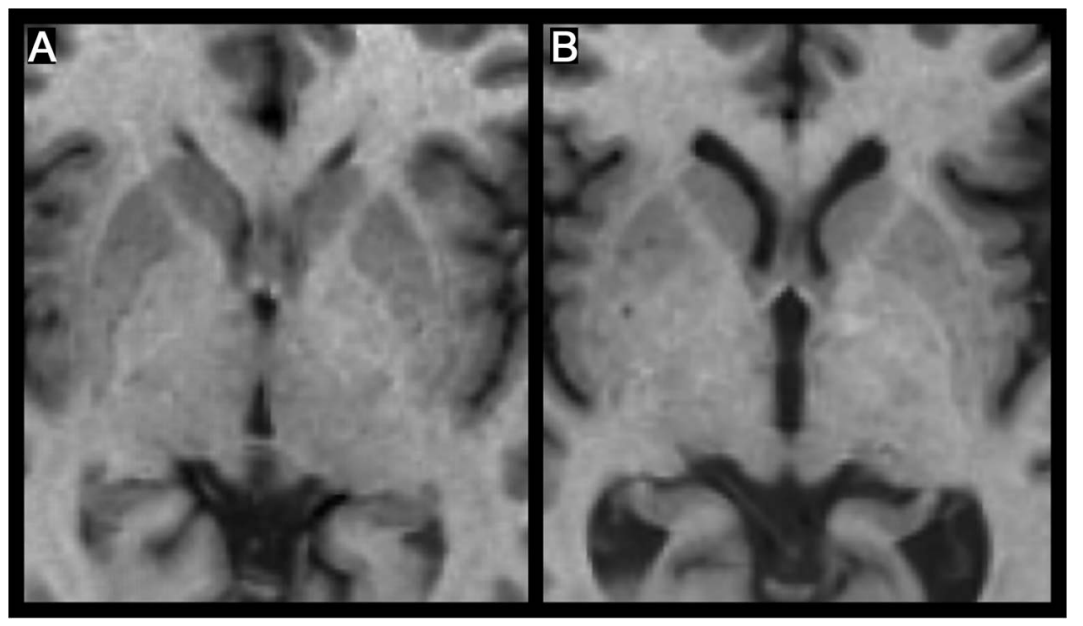

Thalamic atrophy is closely related to third ventricle width, as seen in these images of a patient with relapsing-remitting multiple sclerosis (MS) (A) and a patient with secondary progressive MS (B). Both patients have a disease duration of 14 years, and both images are of the same slice in stereotactic Montreal Neurological Institute 152 space. 
neuronal and axonal degeneration are the likely substrates for accumulating cognitive and motor dysfunction that characterizes long-standing disease. Further research is needed to elucidate the pathologic processes that lead to MRI-detectable thalamic involvement in the early stages in MS and to characterize its role in disease progression. Existing information about thalamic involvement in MS stems mainly from neuropathologic and neuroimaging studies that have a limited number of subjects and contain no clear practical implications for clinicians. Therefore, larger longitudinal studies are required to assess the validity, accuracy, and sensitivity of these preliminary observations. We also recommend that serial measurement of thalamic volume be utilized as a biomarker in MS clinical trials to assess the patient's response to investigational therapeutic agents.

Finally, future neuropathologic MS studies in conjunction with advanced neuroimaging of GM involvement in general, and the thalamus in particular, should include new imaging techniques such as DIR, volumetric inversion recovery T1-based or phase-sensitive sequences, molecular or metabolite imaging, and high-field and ultrahigh-field MRI to further characterize relevant pathogenic mechanisms associated with disease progression over the short and long term.

\section{AUTHOR CONTRIBUTIONS}

Alireza Minagar, Michael H. Barnett, Ralph H.B. Benedict, Daniel Pelletier, Istvan Pirko, Mohamad Ali Sahraian, Elliot Frohman, and Robert Zivadinov substantially contributed to the concept and design of the study. Alireza Minagar, Michael H. Barnett, Ralph H.B. Benedict, and Robert Zivadinov drafted the article, while Daniel Pelletier, Istvan Pirko, Mohamad Ali Sahraian, and Elliott Frohman revised it critically for important intellectual content. All authors had access to the data.

\section{ACKNOWLEDGMENT}

The authors thank Eve Salczynski and Michael G. Dwyer for technical assistance in the preparation of the manuscript.

\section{STUDY FUNDING}

No targeted funding reported.

\section{DISCLOSURES}

A. Minagar reports no disclosures. M. Barnett received personal compensation from Biogen-Idec, Novartis, Merck Serono, and Bayer for speaking and participation in scientific advisory boards. Dr. Barnett's research is supported by Multiple Sclerosis Research Australia and the Nerve Research Foundation, University of Sydney. R.R.H.B. Benedict received research support from Biogen Idec and Shire Inc, and serves on advisory panels for Bayer, Biogen, Novartis, and Actelion. D. Pelletier has received research grant support from Biogen Idec and has provided consulting services to Biogen Idec, Bayer, EMD Serono, Genentech, Synarc Inc., and Teva Neuroscience. I. Pirko serves as Clinical Editor for Nanomedicine: Nanotechnology, Biology and Medicine, and receives research support from the NIH [\#R01NS058698 (PI) and \#R01NS060881 (Co-I)]. M.A. Sahraian has nothing to disclose. E. Frohman has received speaking and consulting fees from Biogen Idec, TEVA Neuroscience, Acorda, Bayer, and Novartis. He has received consulting fees from Biogen Idec, TEVA Neuroscience, Acorda, Novartis, and Abbott Laboratories. R. Zivadinov received personal compensation from Teva Pharmaceuticals, Biogen Idec, EMD Serono, Novartis, and Sanofi-Genzyme for speaking and consultant fees. Dr. Zivadinov received financial support for research activities from Biogen Idec, Teva Pharmaceuticals, Sanofi-Genzyme, Bracco, Questcor Pharmaceuticals, and EMD Serono. Go to Neurology.org for full disclosures.

Received March 29, 2012. Accepted in final form August 6, 2012.

\section{REFERENCES}

1. Kutzelnigg A, Lassmann H. Cortical lesions and brain atrophy in MS. J Neurol Sci 2005;233:55-59.

2. Wegner C, Esiri MM, Chance SA, Palace J, Matthews PM. Neocortical neuronal, synaptic, and glial loss in multiple sclerosis. Neurology 2006;67:960-967.

3. Lucchinetti CF, Popescu BF, Bunyan RF, et al. Inflammatory cortical demyelination in early multiple sclerosis. N Engl J Med 2011;365:2188-2197.

4. Dutta R, Chang A, Doud MK, et al. Demyelination causes synaptic alterations in hippocampi from multiple sclerosis patients. Ann Neurol 2011;69:445-454.

5. Popescu BF, Lucchinetti CF. Meningeal and cortical grey matter pathology in multiple sclerosis. BMC Neurol 2012; 12:11.

6. Hulst HE, Geurts JJ. Gray matter imaging in multiple sclerosis: what have we learned? BMC Neurol 2011;11:153.

7. Cifelli A, Arridge M, Jezzard P, Esiri MM, Palace J, Matthews PM. Thalamic neurodegeneration in multiple sclerosis. Ann Neurol 2002;52:650-653.

8. Vercellino M, Masera S, Lorenzatti M, et al. Demyelination, inflammation, and neurodegeneration in multiple sclerosis deep gray matter. J Neuropathol Exp Neurol 2009;68:489-502.

9. Vercellino M, Plano F, Votta B, Mutani R, Giordana MT, Cavalla P. Grey matter pathology in multiple sclerosis. J Neuropathol Exp Neurol 2005;64:1101-1107.

10. Gilmore CP, Donaldson I, Bo L, Owens T, Lowe J, Evangelou N. Regional variations in the extent and pattern of grey matter demyelination in multiple sclerosis: a comparison between the cerebral cortex, cerebellar cortex, deep grey matter nuclei and the spinal cord. J Neurol Neurosurg Psychiatry 2009;80:182-187.

11. Henry RG, Shieh M, Amirbekian B, Chung S, Okuda DT, Pelletier D. Connecting white matter injury and thalamic atrophy in clinically isolated syndromes. J Neurol Sci 2009;282:61-66.

12. Henry RG, Shieh M, Okuda DT, Evangelista A, GornoTempini ML, Pelletier D. Regional grey matter atrophy in clinically isolated syndromes at presentation. J Neurol Neurosurg Psychiatry 2008;79:1236-1244.

13. Ramasamy DP, Benedict R, Cox JL, et al. Extent of cerebellum, subcortical and cortical atrophy in patients with MS: a case-control study. J Neurol Sci 2009;282:47-54.

14. Calabrese M, Rinaldi F, Mattisi I, et al. The predictive value of gray matter atrophy in clinically isolated syndromes. Neurology 2011;77:257-263.

15. Rocca MA, Mesaros S, Pagani E, Sormani MP, Comi G, Filippi M. Thalamic damage and long-term progression of disability in multiple sclerosis. Radiology 2010;257:463-469.

16. Houtchens MK, Benedict RH, Killiany R, et al. Thalamic atrophy and cognition in multiple sclerosis. Neurology 2007;69:1213-1223.

17. Sullivan EV, Rosenbloom M, Serventi KL, Pfefferbaum A. Effects of age and sex on volumes of the thalamus, pons, and cortex. Neurobiol Aging 2004;25:185-192.

18. Van Der Werf YD, Tisserand DJ, Visser PJ, et al. Thalamic volume predicts performance on tests of cognitive speed and decreases in healthy aging: a magnetic resonance 
imaging-based volumetric analysis. Brain Res Cogn Brain Res 2001;11:377-385.

19. Peterson JW, Bo L, Mork S, Chang A, Trapp BD. Transected neurites, apoptotic neurons, and reduced inflammation in cortical multiple sclerosis lesions. Ann Neurol 2001;50:389-400.

20. Magliozzi R, Columba-Cabezas S, Serafini B, Aloisi F. Intracerebral expression of CXCL13 and BAFF is accompanied by formation of lymphoid follicle-like structures in the meninges of mice with relapsing experimental autoimmune encephalomyelitis. J Neuroimmunol 2004;148:11-23.

21. Magliozzi R, Howell O, Vora A, et al. Meningeal B-cell follicles in secondary progressive multiple sclerosis associate with early onset of disease and severe cortical pathology. Brain 2007;130:1089-1104.

22. Brink BP, Veerhuis R, Breij EC, van der Valk P, Dijkstra CD, Bo L. The pathology of multiple sclerosis is location-dependent: no significant complement activation is detected in purely cortical lesions. J Neuropathol Exp Neurol 2005;64:147-155.

23. Bo L, Vedeler CA, Nyland HI, Trapp BD, Mork SJ. Subpial demyelination in the cerebral cortex of multiple sclerosis patients. J Neuropathol Exp Neurol 2003;62:723-732.

24. van Landeghem FK, Weiss T, von Deimling A. Expression of PACAP and glutamate transporter proteins in satellite oligodendrocytes of the human CNS. Regul Pept 2007; 142:52-59.

25. Evangelou N, Konz D, Esiri MM, Smith S, Palace J, Matthews PM. Size-selective neuronal changes in the anterior optic pathways suggest a differential susceptibility to injury in multiple sclerosis. Brain 2001;124:1813-1820.

26. Pirko I, Lucchinetti CF, Sriram S, Bakshi R. Gray matter involvement in multiple sclerosis. Neurology 2007;68:634-642.

27. Poloni G, Minagar A, Haacke EM, Zivadinov R. Recent developments in imaging of multiple sclerosis. Neurologist 2011;17:185-204.

28. Calabrese M, Rinaldi F, Poretto V, Gallo P. The puzzle of multiple sclerosis: gray matter finds its place. Expert Rev Neurother 2011;11:1565-1568.

29. Seewann A, Kooi EJ, Roosendaal SD, et al. Postmortem verification of MS cortical lesion detection with 3D DIR. Neurology 2012;78:302-308.

30. Geurts JJ, Roosendaal SD, Calabrese M, et al. Consensus recommendations for MS cortical lesion scoring using double inversion recovery MRI. Neurology 2011;76: 418-424.

31. Calabrese M, Atzori M, Bernardi V, et al. Cortical atrophy is relevant in multiple sclerosis at clinical onset. J Neurol 2007;254:1212-1220.

32. Fisniku LK, Altmann DR, Cercignani M, et al. Magnetization transfer ratio abnormalities reflect clinically relevant grey matter damage in multiple sclerosis. Mult Scler 2009; 15:668-677.

33. Audoin B, Zaaraoui W, Reuter F, et al. Atrophy mainly affects the limbic system and the deep grey matter at the first stage of multiple sclerosis. J Neurol Neurosurg Psychiatry 2010;81:690-695.

34. Dalton CM, Chard DT, Davies GR, et al. Early development of multiple sclerosis is associated with progressive grey matter atrophy in patients presenting with clinically isolated syndromes. Brain 2004;127:1101-1107.

35. Ceccarelli A, Rocca MA, Pagani E, et al. A voxel-based morphometry study of grey matter loss in MS patients with different clinical phenotypes. Neuroimage 2008;42: 315-322.

36. Calabrese M, Rinaldi F, Grossi P, et al. Basal ganglia and frontal/parietal cortical atrophy is associated with fatigue in relapsing-remitting multiple sclerosis. Mult Scler 2010;16: $1220-1228$.

37. Mesaros S, Rocca MA, Absinta M, et al. Evidence of thalamic gray matter loss in pediatric multiple sclerosis. Neurology 2008;70:1107-1112.

38. Aubert-Broche B, Fonov V, Ghassemi R, et al. Regional brain atrophy in children with multiple sclerosis. Neuroimage 2011;58:409-415.

39. Kerbrat A, Aubert-Broche B, Fonov V, et al. Reduced head and brain size for age and disproportionately smaller thalami in child-onset MS. Neurology 2012;78:194-201.

40. Till C, Ghassemi R, Aubert-Broche B, et al. MRI correlates of cognitive impairment in childhood-onset multiple sclerosis. Neuropsychology 2011;25:319-332.

41. Hagemeier J, Weinstock-Guttman B, Bergsland N, et al. Iron deposition on SWI-filtered phase in the subcortical deep gray matter of patients with clinically isolated syndrome may precede structure-specific atrophy. AJNR Am J Neuroradiol 2012;33:1596-1601.

42. Bakshi R, Benedict RH, Bermel RA, et al. T2 hypointensity in the deep gray matter of patients with multiple sclerosis: a quantitative magnetic resonance imaging study. Arch Neurol 2002;59:62-68.

43. Ceccarelli A, Filippi M, Neema M, et al. T2 hypointensity in the deep gray matter of patients with benign multiple sclerosis. Mult Scler 2009;15:678-686.

44. Zivadinov R, Heininen-Brown $\mathrm{M}$, Schirda CV, et al. Abnormal subcortical deep-gray matter susceptibilityweighted imaging filtered phase measurements in patients with multiple sclerosis: a case-control study. Neuroimage 2012;59:331-339.

45. Khalil M, Enzinger C, Langkammer C, et al. Quantitative assessment of brain iron by $\mathrm{R}(2)^{*}$ relaxometry in patients with clinically isolated syndrome and relapsing-remitting multiple sclerosis. Mult Scler 2009;15:1048-1054.

46. Reich DS, Smith SA, Gordon-Lipkin EM, et al. Damage to the optic radiation in multiple sclerosis is associated with retinal injury and visual disability. Arch Neurol 2009;66:998-1006.

47. Davies GR, Ramio-Torrenta L, Hadjiprocopis A, et al. Evidence for grey matter MTR abnormality in minimally disabled patients with early relapsing-remitting multiple sclerosis. J Neurol Neurosurg Psychiatry 2004;75:998-1002.

48. Mesaros S, Rocca MA, Pagani E, et al. Thalamic damage predicts the evolution of primary-progressive multiple sclerosis at 5 years. AJNR Am J Neuroradiol 2011;32:10161020.

49. Tovar-Moll F, Evangelou IE, Chiu AW, et al. Thalamic involvement and its impact on clinical disability in patients with multiple sclerosis: a diffusion tensor imaging study at 3T. AJNR Am J Neuroradiol 2009;30:1380-1386.

50. Inglese M, Liu S, Babb JS, Mannon LJ, Grossman RI, Gonen O. Three-dimensional proton spectroscopy of deep gray matter nuclei in relapsing-remitting MS. Neurology 2004;63:170-172.

51. Wylezinska M, Cifelli A, Jezzard P, Palace J, Alecci M, Matthews PM. Thalamic neurodegeneration in relapsingremitting multiple sclerosis. Neurology 2003;60:19491954. 
52. Tellez N, Rio J, Tintore M, Nos C, Galan I, Montalban X Fatigue in multiple sclerosis persists over time: a longitudinal study. J Neurol 2006;253:1466-1470.

53. Tartaglia MC, Narayanan S, Francis SJ, et al. The relationship between diffuse axonal damage and fatigue in multiple sclerosis. Arch Neurol 2004;61:201-207.

54. Leocani L, Colombo B, Comi G. Physiopathology of fatigue in multiple sclerosis. Neurol Sci 2008;29(suppl 2):S241-S243.

55. Minagar A, Sheremata WA, Weiner WJ. Transient movement disorders and multiple sclerosis. Parkinsonism Relat Disord 2002;9:111-113.

56. Zenzola A, De Mari M, De Blasi R, Carella A, Lamberti P. Paroxysmal dystonia with thalamic lesion in multiple sclerosis. Neurol Sci 2001;22:391-394.

57. Benedict RH, Weinstock-Guttman B, Fishman I, Sharma J, Tjoa CW, Bakshi R. Prediction of neuropsychological impairment in multiple sclerosis: comparison of conventional magnetic resonance imaging measures of atrophy and lesion burden. Arch Neurol 2004;61:226-230.

58. Benedict RH, Fischer JS, Archibald CJ, et al. Minimal neuropsychological assessment of MS patients: a consensus approach. Clin Neuropsychol 2002;16:381-397.

59. Batista S, Zivadinov R, Hoogs $M$, et al. Basal ganglia, thalamus and neocortical atrophy predicting slowed cognitive processing in multiple sclerosis. J Neurol 2012;259: 139-146.

60. Mesaros S, Rocca MA, Sormani MP, Charil A, Comi G, Filippi M. Clinical and conventional MRI predictors of disability and brain atrophy accumulation in RRMS: a large scale, short-term follow-up study. J Neurol 2008; 255:1378-1383. 